\title{
Entre o Apoio Matricial e a Atenção Especializada: o Dilema dos Profissionais do Nasf-AB
}

\author{
Between Matrix Support and Specialized Care: \\ The Dilemma of Nasf-AB Professionals
}

\section{RESUMO}

Objetivo: Analisar o discurso produzido por profissionais de saúde das equipes do Núcleo Ampliado de Saúde da Família e Atenção Básica (Nasf-AB) sobre a oferta de cuidados especializados na Atenção Primária à Saúde e suas relações com a organização do sistema de saúde local. Metodologia: Trata-se de uma pesquisa qualitativa na perspectiva teórico-metodológica da análise de discurso de origem francesa, desenvolvida em três municípios da nona região de saúde do Estado da Paraíba, com a participação de 13 profissionais de distintas categorias. Resultados: Do discurso emergiram sentidos que mostram: a) o trabalho das equipes multidisciplinares desenvolvido de modo fragmentado, assistencialista e verticalizado, tendo sua função desvirtuada de apoiadora matricial para reguladora de fluxos para a rede de atenção especializada de baixa densidade; b) a ingerência dos gestores da Atenção Primária à Saúde sobre o trabalho das equipes, suscitando nos profissionais insatisfações sobre o trabalho real desenvolvido e o trabalho prescrito contido nas diretrizes do sistema de saúde brasileiro. Conclusão: Esses profissionais de saúde ainda não conseguiram efetivar seu objetivo de ser apoiador matricial e continuam reproduzindo práticas de cuidado voltadas, prioritariamente, para a cura, a clínica e a especialidade, reafirmando a hegemonia do modelo biomédico na atenção e no cuidado em saúde.

\section{DESCRITORES}

Atenção Primária à Saúde. Profissional da Saúde. Políticas Públicas de Saúde. Pesquisa Qualitativa. Discurso.

\begin{abstract}
Objective: To analyze the discourse presented by health professionals from multidisciplinary teams of the Family Health and Primary Health Care Expanded Support Center (NasfAB) about the provision of specialized care in Primary Health Care and its relations with the organization of the local health system. Methodology: It is a qualitative research based on the Discourse Analysis through a theoretical-methodological perspective developed in three municipalities from the ninth Health Region of Paraíba state, with the participation of 13 health professionals from different categories from Nasf-AB. Results: from the discourse emerged meanings that show: a) the work of these multidisciplinary teams developed in a fragmented, assistentialist, and vertical manner, with their function distorted from matrix support to flow regulator for the low density, specialized care network; $b$ ) the intervention of Primary Health Care managers on the work of these teams, causing dissatisfaction among professionals about the real work developed and the prescribed work contained in the guidelines of the Brazilian health system. Conclusion: these health professionals have not yet achieved their goal of being a matrix supporter and continue to reproduce the care practices focused primarily on healing, clinic, and specialty, reaffirming the hegemony of the biomedical model in health care.
\end{abstract}

\section{DESCRIPTORS}

Primary Health Care. Health Personnel. Health Policy. Qualitative Research. Discourse.

${ }^{1}$ Mestre em Saúde da Família pelo Programa de Pós-Graduação Mestrado Profissional em Saúde da Família. Universidade Federal da Paraíba, João Pessoa, Paraíba, Brasil.

2 Doutor em Saúde Pública pela Universidade Federal da Bahia. Professor Adjunto no Departamento de Fisioterapia e dos Programas de Pós-Graduação em Saúde Coletiva e Fisioterapia do Centro de Ciências da Saúde da Universidade Federal da Paraíba, João Pessoa, Paraíba, Brasil.

${ }^{3}$ Doutor em Saúde Pública pelo Instituto de Pesquisas Aggeu Magalhães - FIOCRUZ/PE. Professor Adjunto do Departamento de Fisioterapia e dos Programas de Pós-Graduação em Saúde da Família, Saúde Coletiva e Fisioterapia do Centro de Ciências da Saúde da Universidade Federal da Paraíba, João Pessoa, Paraíba, Brasil.

${ }^{4}$ Doutora em Educação pela Universidade Federal da Paraíba. Professora Associada do Departamento de Fisioterapia e dos Programas de Pós-Graduação em Modelos de Decisão e Saúde do Centro de Ciências Exatas e da Natureza da Universidade Federal da Paraíba e Fisioterapia do Centro de Ciências da Saúde da Universidade Federal da Paraíba, João Pessoa, Paraíba, Brasil.

${ }^{5}$ Doutora em Ciência da Saúde pela Universidade Federal do Rio Grande do Norte. Professora do Departamento de Enfermagem e do Programa de Pós-Graduação em Saúde Pública da Universidade Estadual da Paraíba. Campina Grande, Paraíba, Brasil.

${ }^{6}$ Doutora em Saúde Coletiva pela Universidade Federal da Bahia. Professora Associada III do Departamento de Medicina Preventiva e Social da Faculdade de Medicina da Bahia da Universidade Federal da Bahia e Docente Permanente do Programa de Pós-Graduação em Saúde, Ambiente e Trabalho (PPGSAT), Salvador, Bahia, Brasil. 
$\mathrm{O}_{\mathrm{s}}^{\mathrm{N}}$ Núcleo Ampliado de Saúde da Família e Atenção Básica (Nasf-AB), denominado em sua criação de Núcleo de Apoio à Saúde da Família (NASF), surgiu em 2008 como uma importante estratégia de atenção para ampliar e qualificar o escopo de ações de saúde das equipes de Atenção Primária à Saúde (APS) brasileiras, por meio da inserção de diferentes categorias profissionais na APS ${ }^{1}$.

Orientado pelo referencial teóricometodológico do Apoio Matricial de Campos $^{2}$ o Nasf-AB presta retaguarda clínicoassistencial e técnico-pedagógico as equipes de APS prezando por construções singulares, diálogo, decisão compartilhada, responsabilização e pactos assumidos entre equipe de referência e de apoio sem assumir a referência do cuidado ou se caracterizando como um segundo nível de atenção para o qual o usuário possa ser encaminhado ${ }^{3}$.

A utilização do termo matricial aponta para a mudança de posição do especialista em relação ao profissional que demanda seu apoio, de modo a romper com a verticalização das relações que mantém uma diferença de autoridade e transferência de responsabilidade entre aquele que encaminha e quem o recebe, característico dos tradicionais serviços de saúde. Assim, a ideia de matriz é manter uma relação horizontal entre os profissionais de referência e os apoiadores com base em processos dialógicos ${ }^{4}$.

Entretanto, nem sempre o processo de trabalho no Nasf-AB está fundamentando com base no apoio matricial, até porque os documentos ministeriais que instituíram a proposta em 2008 deixaram a interpretação sobre sua organização e papel a critério do contexto político e especificidades da rede de saúde local, permitindo a coexistência de diferentes modelos de operacionalização ${ }^{5,6}$.

Nascimento et al. ${ }^{7}$ sistematizam três tipos de configurações do Nasf-AB de acordo com sua aproximação com o modelo ideal de apoio matricial: Equipe matricial que segue o preconizado nos documentos de implantação do Nasf-AB; Equipe semimatricial, com priorização do atendimento individual em detrimento de ações conjuntas; e Equipe assistencial-curativista, cujo apoio é apenas um acesso a especialistas.

A introdução do Nasf-AB na APS mostra-se complexa uma vez que tanto os profissionais de saúde quanto a sociedade civil ainda os entendem conforme a construção histórica de suas profissões e desconhecem o apoio matricial como ferramenta para potencializar a $\mathrm{APS}^{8}$. Além disso, a desestruturação das Redes de Atenção à Saúde (RAS) e a grande demanda reprimida para a média complexidade evidenciam percalços relacionados ao contexto estrutural de trabalho que marca uma grande pressão pelos atendimentos clínicos das especialidades que não estão sendo forne-cidos nos outros níveis de atenção e acabam por exigir do Nasf-AB respostas clínicas-assistenciais $^{9,10}$ delineadas por cada profissional frente à realidade que encontram ${ }^{11-13}$.

Tendo em vista que as distintas apresentações do Nasf-AB impactam na ordenação do seu processo de trabalho e na prestação de cuidados no contexto da APS e, por vez, escondem os reais problemas a serem enfrentados na oferta de serviços especializados à população, esse estudo visa analisar o discurso produzido pelos 
profissionais de saúde do Nasf-AB sobre a oferta de cuidado especializado e suas relações com a organização do sistema de saúde local.

\section{METODOLOGIA}

Trata-se de uma pesquisa qualitativa combasenaperspectivateórico-metodológica da análise de discurso (AD) de origem francesa14, desenvolvida na $9^{a}$ Região de Saúde do Estado da Paraíba, segundo os propósitos do Mestrado Profissional em Saúde da Família de investigar situações ligadas a prática do profissional de saúde.

O cenário envolveu os 15 municípios da região que possuíam cobertura de 74 equipes de saúde da família (eSF) e 17 equipes do Nasf-AB (EqNasf-AB). Para participar da pesquisa os profissionais das EqNasf-AB deveriam estar vinculados a equipe por meio do Cadastro Nacional de Estabelecimento de Saúde e não exercer outro cargo como gerência, chefia ou confiança. As equipes foram abordadas até que se alcançou 0 critério de saturação teórica ${ }^{15}$, que ocorreu após a $5^{\mathrm{a}}$ equipe no $3^{\circ}$ município.

Em um primeiro momento contactouse via telefone a coordenação das EqNasf$A B$ para apresentar o estudo e agendar um momento de diálogo com os profissionais constituintes para esclarecer seus objetivos e realizar o convite a colaboração conforme disponibilidade. Não ocorreram recusas em participar da pesquisa por aqueles que estavam presente na ocasião, totalizando 13 participantes.

A coleta de dados foi realizada por uma pesquisadora devidamente treinada e ocorreu entre os meses de novembro de
2018 a fevereiro de 2019 no local acordado com cada entrevistado. O material empírico foi obtido mediante a técnica de entrevista semiestruturada ${ }^{16}$, norteada por um roteiro pré-estabelecido elaborado e testado originalmente para este fim.

Cada entrevista foi gravada, transcrita para o meio digital e, posteriormente, analisada por meio da $A D$ em três perguntas heurísticas: Qual é o conceito-análise presente no texto? Como o texto constrói o conceito-análise? A que discurso pertence o conceito-análise construído da forma que o texto constrói? ${ }^{17}$.

Inicialmente realizou-se a leitura flutuante do material para identificação dos primeiros pressupostos da análise. Em seguida, efetuou-se a leitura analítica do texto com aplicação da primeira pergunta heurística, definindo-se o conceito-análise a posteriori: trabalho no Nasf-AB. Ao deferir a segunda pergunta heurística, observouse que as marcas textuais construíam o conceito-análise a partir de três subdivisões: 1) trabalho em equipe; 2) fluxo de acesso; e 3) processo de trabalho.

Considerou-se como marcas textuais as paráfrases (o que se mantém), a polissemia (deslocamento), a metáfora (transferência), a relação do dizer e não dizer (silêncio) e as modalizações (suavizações), destacandose também as condições de produção que representam o contexto de elaboração do discurso ${ }^{14}$. Para finalizar, fez-se a terceira pergunta heurística a fim de identificar o discurso do conceito-análise construído e os sentidos produzidos.

O projeto foi aprovado pelo Comitê de Ética em Pesquisa conforme Parecer consubstanciado $\mathrm{n}^{\circ}$. 2.677.650. Todos os procedimentos éticos envolvendo pesquisas 
com seres humanos foram respeitados incluindo a participação voluntária na pesquisa, após a leitura e a assinatura do Termo de Consentimento Livre e Esclarecido. Para preservar o anonimato dos participantes, os recortes transcritos das falas foram identificados pelas iniciais de acordo com a categoria profissional e seguidos por números cardinais conforme ordem de entrevista.

\section{RESULTADOS E DISCUSSÃO}

Condições de produção

$O$ arranjo das EqNasf-AB é definido conforme a organização municipal de saúde, podendo ser composta por 19 categorias de saúde distintas ${ }^{1}$ representadas nesse estudo por: assistente social, fisioterapeuta, fonoaudiólogo, nutricionista e psicólogo, com tempo de trabalho no Nasf-AB, mínimo de 1 ano e 8 meses e máximo de aproximadamente 7 anos até o momento da coleta de dados. Do total, 7 eram mulheres e 6 homens com idade que variou entre 25 e 58 anos e tempo de graduado de 2 a 31 anos; 9 formados em instituições de ensino privadas e 6 possuíam pós-graduação em área correlata a saúde coletiva.

O trabalho em equipe no Nasf-AB - um cuidado fragmentado

$O$ trabalho em equipe desenvolvido por essas EqNasf-AB foi marcado pelo conhecimento especializado determinando a atuação fragmentada de cada componente: "cada profissional ele trabalha" (Ps3); "cada profissional [...] vai atender a sua demanda" (As1); "cada um com sua especialidade" (Fo1); "a gente trabalha mais individual do que como equipe" (Fo1).

A diferença entre o trabalho multiprofissional e o interprofissional é posta na díade fragmentação e integralidade. No primeiro as ações são paralelas, justapostas e centradas em profissionais específicos; enquanto no segundo existe articulação e integração entre profissionais de modo a superar uma atuação parcelada ${ }^{18}$.

Agrupar os profissionais em equipe não necessariamente se traduz em uma prática colaborativa, pois, a cooperação interprofissional envolve um constante processo de comunicação entre os profissionais da equipe de saúde para a tomada de decisão, permitindo uma atuação convergente com os diferentes conhecimentos e habilidades dos núcleos profissionais, usuário e comunidade ${ }^{9}$.

Os achados encontrados no estudo em tela corroboram com a literatura que aponta os profissionais do Nasf$A B$ organizando seu trabalho a partir da centralidade de seu núcleo profissional, fragmentando o cuidado prestado ${ }^{19,20}$. Neste sentido, resta entender para quê ou a que este tipo de modelagem está servindo?

Fluxo de Acesso ao Nasf-AB As demandas que chegam - uma porta aberta

OfluxodeacessoaoNasf-ABapontou que as demandas são referenciadas, encaminhadas ou recebidas pelo Nasf$A B$, caracterizando-o como um serviço 
porta aberta na rede: "as demandas que vêm das Unidades de Saúde" (Ps3); "referenciado pela UBS [Unidade Básica de Saúde], e pelos outros setores também como ação social, educação na escola [...] de outros municípios [...] São as pessoas que encaminham pra cá" (Ps4); "a gente acaba que meio que recebendo é... pacientes diretamente no próprio serviço" (Fi3); "chega de forma espontânea" (As2).

O encaminhamento diretamente para o Nasf realizado por alguém e sem passar pela UBS também foi realidade num estudo conduzido com usuários em município de médio porte de Minas Gerais, no qual os autores enfatizaram que o Nasf não é porta de entrada e que o fluxo de acesso deve ser proveniente da unidade de saúde local para não fragmentar o cuidado ofertado. Entretanto, a própria deficiência de compreensão do que o Nasf representava e a falta de divulgação sobre suas ações, especialmente pelos profissionais das equipes de APS, geravam equívocos sobre o acesso a esses especialistas e, consequentemente, nas percepções dos usuários que ambos os serviços não apresentavam articulação ${ }^{21}$.

Os documentos que instituíram o Nasf-AB definem que o mesmo deve atuar de forma integrada a RAS, a partir das demandas identificadas em parceria com as equipes de APS, ampliando o acompanhamento longitudinal da eR e fortalecendo a coordenação do cuidado com responsabilização compartilhada entre as equipes na prática dos encaminhamentos, desconstruindo a lógica da referência e contrarreferência existente ${ }^{22}$.
Assim, o Nasf parece servir a uma lógica que se assemelha mais a um serviço ambulatorial da média complexidade que burla as centrais de marcação e, por conseguinte, mascara o afogamento do aparelho de regulação para esses serviços.

A tomada de decisão - um regulador de fluxos de acesso

Os profissionais do Nasf-AB avaliam as demandas que recebem e realizam encaminhamentos conforme necessidade, funcionando como um equipamento regulador na rede: "faz esse atendimento [...] se [...] a pessoa necessita de um atendimento mais especializado [...] eu vou e encaminho" (Fo1); "atuamos também como mediadores das unidades de saúdes pra os serviços de cunho secundário e terciário [...] a gente faz esse intermédio, né?" (Fi3); "a proposta do NASF é justamente fazer essa mediação entre o serviço básico e o serviço mais especializado" (Fi3); "a gente faz esse intercâmbio pra manter o processo de trabalho fluindo" (As2).

O Nasf-AB pode assumir o papel de articulador entre equipe de APS e os demais serviços da rede intra e intersetorial a fim de estabelecer fluxos para garantir a integralidade do cuidado na $\mathrm{RAS}^{13}$. No entanto, os discursos aqui mostram o Nasf-AB recebendo e encaminhando as demandas de forma desarticulada com a eR, reproduzindo de forma verticalizada a referência e contrarreferência. Ademais, o Nasf-AB aparece como um nível de atenção intermediário entre 
a APS e atenção especializada, ora atuando como porta aberta recebendo demandas sem necessidade de origem, ora servindo como antessala da atenção especializada, realizando os primeiros cuidados especializados e regulando os fluxos para esse nível de atenção sem negociação a priori.

Embora exista uma preocupação dos profissionais do Nasf e da equipe de APS a respeito de sua relação com diferentes serviços, por vezes, esse diálogo ainda é restrito apenas a encaminhamentos particularizados por área, onde trocas e ações estão adstritas aos profissionais de acordo com seu núcleo de saber ${ }^{19}$. Além disso, os resultados distinguem outra função subliminar do Nasf-AB, qual seja, a de retirar dos cuidados da eR os usuários os quais tencionam as equipes diariamente com sua demanda reprimida de cuidados longitudinais de média complexidade e que não conseguem devido à baixa disponibilidade de serviços.

\section{O processo de trabalho no Nasf-AB}

Integração equipe de referência e de apoio - Relações verticais

As equipes de APS demandam apoio do Nasf-AB quando veem a necessidade de uma intervenção específica, mediante ficha de referência e contrarreferência ou solicitação direta ao profissional especialista, assinalando uma relação vertical entre as equipes: "a gente se organiza é através de demandas que vêm das unidades de saúde, e... através das referências que nos são repassadas, né?!" (As1); "A gente se organiza de acordo com a demanda que chega, tá?" (Nu1); "quando nos é solicitado" (As1); "e outras demandas que a enfermeira vier a solicitar ou as... os demais componentes da equipe de Saúde da Família” (Ps1).

Outrossim, são apresentadas modalizações do discurso com momentos de articulação entre a eSF e o Nasf-AB: "reunião com a equipe de referência, né?" (As2); "fazer uma breve discussão [...] com o profissional que tá me repassando, certo?" (As1) que com uso das interrogações "né?", "certo?" reforçam a tentativa do enunciador de obter concordância e suavizar o sentido de atuação das equipes de maneira independente.

Agregar outras categorias profissionais na APS por meio do Nasf-AB tem proporcionado benefícios relacionados à ampliação do cuidado ofertado pela eSF, embora, ainda, persistam as visões de trabalho individualizado e pouco integrado entre equipes que diante de determinados casos tendem a encaminhar o usuário ao invés de buscar a equipe de apoio na cogestão do cuidado ${ }^{\text {. }}$.

Embora algumas EqNasf-AB desejarem um trabalho integrado com as eSF, para outras essa relação ainda é frágil e inexistente, por motivos que vão da falta do reconhecimento do Nasf enquanto apoiador matricial, a própria resistência das eSF em atuar sob essa lógica ${ }^{7}$.

Os resultados aqui encontrados corroboram com essas discussões, trazendo a conexão entre encaminhamentos e verticalização das relações entre pro- 
fissionais de referência e de apoio ainda não superada, reproduzindo o cuidado fragmentado, individualizado e independente entre essas equipes na APS que reforçam um modelo de operação do Nasf-AB como rota de fuga para a sobrecarregada da eSF e para a ineficiente média complexidade.

Atuação do Nasf-AB - Ambulatório de especialidades

O trabalho desenvolvido pelo Nasf-AB envolve atividades de cunho pedagógico e assistencial: "ações dentro das unidades" (Fi1); "realização de palestras" (Fi3); "salas de esperas" (As1); "um Novembro Azul, um Outubro Rosa, alguma coisa de Setembro Amarelo" (As1); "Programa de Saúde na Escola" (Ps3); "hiperdias nas UBS" (Fi3); "atendimento na própria Unidade Básica de Saúde" (Fi3); "atendimento coletivo" (Ps3); "fazendo acompanhamento de algum caso" (Fi3); "debate sobre casos clínicos" (Fi3); "campanhas" (Fo1); "visitas domiciliares" (Fo1); "em parceria com os outros serviços, né? CRAS, CREAS, a creche [...] as demais da rede de serviços a gente também atua" (Ps1).

No entanto, marcas textuais mostram que as atividades assistenciais de saúde em uma modalidade de ambulatório estão sobrepostas as demais: "a gente é assim colocado mais nessa parte ambulatorial" (Fi2); "o atendimento é realizado [...] numa modalidade de ambulatório" (Ps1); "a maioria das demandas que chegam na Atenção Básica, é essa demanda ambulatorial" (Ps2).
A utilização da palavra ambulatório ao invés de atendimento individual específico corrobora para o olhar especializado dos sujeitos sobre o seu trabalho, visto que esse vocábulo remete a memória discursiva de um atendimento clínico realizado pelo especialista dentro do consultório, que difere da proposta do Nasf-AB enquanto apoio especializado, mas não ambulatório de especialidades ${ }^{23}$. Desse modo, o sentido construído é da atuação do Nasf-AB com foco nas atividades assistenciais sobre as demais ações de saúde, pautado no discurso do assistencialismo, distanciando-se do seu propósito de origem.

Uma vez que o papel do especialista é supervalorizado em detrimento ao de apoiador matricial, suscita excesso de demandas assistenciais para esse núcleo como constatado em outros estudos ${ }^{7,11,24}$.

A proposta do Nasf-AB é reorientar o modelo de atenção à saúde tradicional, centrado na assistência curativa, individual, fragmentada, verticalizada e medicalizadora, avançando na corresponsabilização e cogestão do cuidado $^{5,10}$. No entanto, os profissionais do Nasf-AB continuam reproduzindo as práticas do modelo de atenção à saúde que se propõem superar,6,9,13.

Essa assimilação ou continuidade da identidade de especialista expõe o fato de que os profissionais que estão no Nasf-AB também contribuem para o modelo tácito que se configura para este núcleo, o qual se distancia da proposta de apoio matricial e serve como cortina de fumaça para o hiato deixado pela média complexidade. 
Insatisfações com o processo de trabalho no Nasf-AB - "o porquê é"

Os dizeres dos profissionais do Nasf-AB sinalizaram também para uma insatisfação com seu processo de trabalho: "[O processo de trabalho] É um poucooo solto, né?!" (Fo1); "é algo assim, meio "alheio" [...] não há uma sistematização nessa organização do processo de trabalho, é algo que dificulta muito" (As1); "precisava de um suporte logístico para os profissionais desenvolverem suas habilidades técnicas" (Fi1); "precisaríamos mais, também, [...] equipamentos para trabalhar a promoção de saúde, palestras educativas" (Fi1); "a gente não disponibiliza de um carro próprio, pra gente fazer as visitas" (As1); "como não tem carro [...] a atuação e o atendimento é realizado na policlínica, numa modalidade de ambulatório" (Ps1); "A minha equipe tá bastante desfalcada" (Fi2); "eu tô me sobrecarregando enquanto de repente se contrata, né?" (Ps2); "os contratados não cumprem [horário] e... a questão salarial é bem diferente" (Ps1); "muitos [contratados] não vêm para o trabalho, não vindo pra o trabalho sobrecarrega as demandas" (Ps2); "a gente tem um pouco de uma visão meio que deturbada de algumas Unidades Básicas de Saúde que acham que o serviço do NASF é um serviço mais de cunho terciário" (Fi3).

Tais descontentamentos estão voltados para falta de organização do processo de trabalho, necessidade de recursos humanos e materiais para propiciar uma melhor execução das atividades e, também, insuficiência de conhecimentos sobre o papel do NasfAB. Além disso, existem elementos textuais que demonstram sentimentos ambíguos concernentes ao vínculo trabalhista sob o regime de contrato do profissional do Nasf-AB. Se por um lado o profissional contratado pode ter mais privilégios que o concursado, por outro, esse tipo de vínculo é instável levando-o a subordinação ainda que em situação desfavoráveis, como visto no fragmento textual abaixo:

[...] a gente foge um pouquinho da competência do NASF, a gente acaba fazendo mais ambulatorial. Nem sempre tem um diálogo também com as enfermeiras, com as agentes de saúde, certo?! Então a gente é assim colocado mais nessa parte ambulatorial, até mesmo porque todos que compõem o NASF são contratados [...] e isso acaba, assim... é... a gente tem que acatar né?! [...] então a gente foge um pouquinho da competência do NASF geralmente pra suprir algumas coisas aí de... Ordens né?! [...] (Fi2).

Esse sujeito deixa evidente em sua fala que existe uma "competência do NASF" que parte do princípio de uma organização do processo de trabalho com integração entre eSF e Nasf-AB a qual é desviada para cumprir ordens de manter seu trabalho focalizado no atendimento ambulatorial.

Dessa maneira, o discurso expressa 
que existe uma relação de poder da gestão enquanto órgão responsável pela execução do Nasf-AB sobre os profissionais que compõem as equipes, com submissão desses a distintas condições de trabalho que foge da sua autonomia ou desviam do seu propósito, gerando os sentimentos de insatisfação com o processo de trabalho.

Justificativas para $\mathrm{o}$ trabalho no Nasf-AB não ser efetuado conforme disposto nos documentos oficiais, giram em torno da relação com a gestão, eSF e usuários. A gestão por não apoiar as EqNasf-AB no planejamento e suporte de recursos adequados; as eSF que por terem dificuldade de reconhecer e aceitar o trabalho do Nasf-AB e demandar apoio sob lógica assistencialista e curativista; e os usuários por valorizarem o cuidado focado em procedimentos ${ }^{25}$.

A tendência do Nasf-AB em reproduzir práticas assistenciais no cuidado reforça a permanência de um modelo de atenção à saúde ainda centrada na clínica e cura. Entre os elementos que levam a essa predisposição estão a falta de apoio da gestão e a manutenção de condições de trabalhos que impossibilitam a reorganização das práticas no Nasf, afetando sua articulação com as eSF e fortalecendo o modelo médico-assistencial-privatista do cuidado individual em detrimento da sua principal função: apoiador matricial ${ }^{10}$.

Ausência de planejamento dos municípios para efetivarem a proposta do Nasf-AB assinala que muitas vezes estiveram mais preocupados em receber o recurso do Ministério da Saúde sem, contudo, investir de maneira política, técnica e financeira no programa ${ }^{10}$. Outrossim, a gestão municipal assume um papel de defensora do modelo de atenção à saúde médico-assistencial-privatista ao especificar a operacionalização do Nasf$A B$ que pretende implantar ${ }^{5}$. Mais que isso, é possível inferir que a gestão ao não investir também na média complexidade coloca sobre os ombros das EqNasf-AB o fardo de dar conta das demandas próprias da assistência do nível secundário.

A competência do Nasf-AB - "o como deveria ser"

Os sujeitos expõem insatisfações com o processo de trabalho por possuírem conhecimentos prévios sobre o papel do Nasf-AB - a "competência do NASF", e evocam os documentos de sua institucionalização para sustentar - seu dizer: "a cartilha do NASF" (Ps2); o "caderno de diretrizes" (Fi3), o "protocolo" (Fi3). Assim, começam a ser construídos os sentidos do "como deveria ser" o processo de trabalho no Nasf-AB: "um trabalho educativo mais do que atendimento ambulatorial" (Nu1); "um trabalho precoce mesmo, de NASF" (Fo1); "atendimento individual quando necessário" (Ps2); "atendimento compartilhado seja com os profissionais do NASF [...] ou seja com os profissionais da Unidade Básica de Saúde" (Ps2); "os grupos" (Ps2); "orientando também a equipe da Unidade Básica de Saúde [...] Como intervir, como encaminhar SE NECESSÁRIO pra um serviço especializado. Quando chamar a gente 
[Nasf-AB] nesse sentido" (Ps2); "planejar, organizar, realizar projetos terapêuticos singulares, né? Ver realmente como é que tá a situação, fazer um diagnóstico territorial" (Fi3); "o NASF ele não serve como porta de entrada pra o usuário e sim como uma... uma mediação entre setores" (Fi3); "articulação às vezes com a rede e com o serviço que é do NASF" (Ps2).

O trabalho do Nasf-AB deveria então priorizar atividades de promoção/ prevenção da saúde, mas também realizar atividades assistenciais individuais e compartilhadas desde que existisse integração com as eSF para definição de fluxos e articulação com demais pontos da RAS.

Esse discurso de conhecimento prévio tenta modalizar os sentidos construídos sobre o trabalho no Nasf$A B$ hegemonicamente: fragmentado, assistencialista, verticalizado e porta aberta; ratifica o sentido de regulador de fluxos; e não intervém no sentido de submisso, visto que continuam dependendo da gestão para execução do seu trabalho mesmo que conforme recomendações ministeriais.

O desafio do vir a ser também se mostrava no cotidiano dos profissionais de saúde do Nasf do estudo realizado em São Paulo-SP, uma vez que sabiam o que não deveriam ser e estavam prontos para executar o seu papel, mas conviviam com obstáculos que impediam que isso acontecesse ${ }^{12}$.

Outro estudo trouxe o discurso do Nasf apontando um deve ser voltado para o trabalho em equipe e clínica ampliada, corroborando com os pressupostos dos documentos oficiais de institucionalização do programa. Apesar disso, concretizar o deve ser em fazer acontecer ainda se mostrou longínquo, visto que o trabalho se apresenta fragmentado, focado na assistência, e com desvalorização dos espaços de reuniões entre equipes de saúde para pactuação de ações ${ }^{25}$.

Nesse estudo o como deveria ser corrobora com a literatura onde existe um hiato entre o trabalho prescrito e o trabalho real no Nasf-AB em decorrência de vários desafios no cotidiano dos serviços de saúde, distanciando-o das diretrizes propostas ${ }^{11,13}$. À vista disso, o trabalho prescrito para o Nasf-AB não é o estabelecido nos documentos (apoiador matricial), mas sim um trabalho ambulatorial ditado por uma composição de forças (gestão, eSF, usuários e, em alguma medida, pelos próprios profissionais) mediadas por duas situações: a carência de serviços de cunho especializados e a necessidade de desafogar as $\mathrm{eR}$ das demandas não respondidas da atenção secundária ${ }^{9,10}$.

O acesso à média complexidade é tido como gargalo, buraco e um dos maiores obstáculos do SUS ${ }^{26}$. O desafio na garantia de ascensão a esse nível de atenção deriva do modelo de atenção à saúde adotado, a capacidade resolutiva da APS, o dimensionamento e a organização da disponibilidade dos serviços ${ }^{27}$.

$\mathrm{Na}$ ausência dessa rede de suporte especializado, a sociedade que valoriza o modelo médico-assistencial-privatista no cuidado em saúde tende a demandar do Nasf-AB que abarque as demandas 
ambulatoriais de acordo com suas categorias profissionais. Para isso, não precisa existir diálogo horizontal entre equipes de referência e apoio, apenas, o simples encaminhamento do profissional da APS ao núcleo de conhecimento que assenta dar resposta ao problema clínico identificado. Dessa maneira, o Nasf$A B$ passa a ser uma panaceia da APS, com atribuições explícitas de remediar o trabalho especializado, uma vez que o sistema possui problemas estruturais para suprir essas demandas.

Tendo em vista que a própria resolutividade da APS depende, em parte, da disponibilidade dos serviços especializados e tecnológicos, a integralidade e a longitudinalidade do cuidado no SUS, coordenada pela APS, torna-se inviável ${ }^{25} \mathrm{e}$, assim, os profissionais de saúde do saúde do Nasf-AB produzem um discurso de equipe semimatricial que prioriza o assistencialismo na sua prática ainda que desenvolva outras atividades ${ }^{7}$ por serem delegados a assumir posição de cuidado especializado e maquiar na APS a resolutividade dos casos que na realidade deveriam estar em acompanhamento no segundo nível de atenção à saúde.

\section{CONCLUSÃO}

O discurso dos profissionais de saúde do Nasf-AB hegemonicamente apontou para profissionais de saúde que atuam com foco no cuidado individual e assistencial conforme cada especialidade, fragmentando o trabalho em equipe e assumindo função de nível secundário de atenção à saúde que mantém uma relação vertical com a APS. A gestão determina a configuração e as condições de trabalho no Nasf-AB o que, consequentemente, gera insatisfações nesses profissionais a respeito do processo de trabalho imposto, visto que estes têm ciência da portaria do Nasf-AB e seu norte para atuação dos especialistas-apoiadores na APS.

A fragilidade entre o trabalho prescrito e o trabalho real no Nasf-AB ressalta a carência de uma rede estruturada de serviços de média complexidade e as dificuldades da reorientação do modelo de atenção à saúde no país que prezam ainda por uma cultura assistencial, fragmentada em especialidades e com diálogo vertical entre profissionais; com poucos avanços no apoio matricial enquanto reorganizador do trabalho em saúde por meio do diálogo horizontal, a decisão compartilhada, a corresponsabilização e a cogestão do cuidado na APS.

Nesse estudo apenas os profissionais do Nasf-AB foram ouvidos, por isso, ulteriores pesquisas que proponham analisar o discurso da gestão, eSF e usuários sobre o trabalho do Nasf-AB são recomendados para melhor compreender as potencialidades e impasses da execução desse serviço na APS sob novos olhares, até porque outros fatores podem estar intervindo no trabalho do Nasf$A B$, como a resistência dos profissionais das equipes de referência e apoio a novas práticas de saúde tal qual apoio matricial, ou a própria gestão e população que ainda não o compreende, carecendo de estudos que se debrucem sobre essas questões 


\section{REFERÊNCIAS}

1. Ministério da Saúde. Portaria $n^{\circ} 2.436$, de 21 de setembro de 2017. Aprova a Política Nacional de Atenção Básica, estabelecendo a revisão de diretrizes para a organização da Atenção Básica, no âmbito do Sistema Único de Saúde (SUS). Diário Oficial da União 2017; 21 set.

2. Campos GWS. Equipes de referência e apoio especializado matricial: um ensaio sobre a reorganização do trabalho em saúde. Ciênc Saúde Coletiva 1999; 4:393-403.

3. Castro C, Campos GWS. Apoio Matricial como articulador das relações interprofissionais entre serviços especializados e atenção primária à saúde. Physis (Rio J.) $2016 ; 26: 455-481$.

4. Campos GWS, Domitti AC. Apoio matricial e equipe de referência: uma metodologia para gestão do trabalho interdisciplinar em saúde. Cad Saúde Pública 2007; 23:399-407.

5. Sampaio J, Sousa CSM, Marcolino EC, Magalhães FC, Souza FF, Rocha AMO, et al. O NASF como dispositivo da gestão: limites e possibilidades. Rev Bras Ciênc Saúde 2012; 16:317-324.

6. Arce VAR, Teixeira CF. Práticas de saúde e modelo de atenção no âmbito do Núcleo de Apoio à Saúde da Família em Salvador (BA). Saúde Debate 2017; 41:228-240.

7. Nascimento CMB, Albuquerque PC, Sousa FOS, Albuquerque LC, Gurgel IGD. Configurações do processo de trabalho em núcleos de apoio à saúde da família e o cuidado integral. Trab Educ saúde. 2018; 16:1135-56.

8. Arce VAR. Núcleos de Apoio a Saúde da Família: uma análise das práticas de saúde e do processo de construção da identidade profissional no contexto de Salvador, BA [tese] Salvador (BA): Universidade Federal da Bahia; 2016.

9. Matuda CG, Pinto NR, Martins CL, Frazão P. Colaboração interprofissional na Estratégia Saúde da Família: implicações para a produção do cuidado e a gestão do trabalho. Ciênc. Saúde Colet. 2015; 20:2511-21.

10. Arce VAR, Teixeira CF. Atividades desenvolvidas por profissionais de núcleos de apoio à saúde da família: revisão da literatura. Trab Educ Saúde. 2018; 16:1443-64.

11. Gonçalves RMA, Lancman S, Sznelwar LA, Cordone NG, Barros JO. Estudo do trabalho em Núcleos de Apoio à Saúde da Família (NASF), São Paulo, Brasil. Rev Bras Saúde Ocup. 2015; 40:59-74.

12. Lancman S, Gonçalves RMA, Cordone NG, Barros JO. Estudo do trabalho e do trabalhar no Núcleo de Apoio à Saúde da Família. Rev Saúde Pública 2013; 47:968-75.

13. Macedo MAV, Ximenes-Guimarães JM, Coelho-Sampaio JJ, Pereira-Morais AP, Carneiro C. Análise do processo de trabalho no núcleo de apoio à saúde da família em município do nordeste brasileiro. Rev Gerenc Políticas Salud. 2016; 15:194-211.

14. Orlandi EP. Análise de Discurso: princípios \& procedimentos. 13 ed. Campinas: Pontes; 2020.
15. Falqueto JMZ, Hoffmann VE, Farias JS. Saturação teórica em pesquisas qualitativas: relato de uma experiência de aplicação em estudo na área de administração. Rev ciênc Administração. 2018; 20:40-53.

16. Minayo MCS, Costa AP. Fundamentos teóricos das técnicas de investigação qualitativa. Rev Lusófona de Educação. 2018; 40:139-153.

17. Souza SAF. Análise de discurso: procedimentos metodológicos. Manaus: Instituto Census; 2014.

18. Barros NFD, Spadacio C, Costa MVD. Trabalho interprofissional e as Práticas Integrativas e Complementares no contexto da Atenção Primária à Saúde: potenciais e desafios. Saúde Debate. 2018; 42:163-173.

19. Ferro LF, Silva EC, Zimmermann AB, Castanharo RCT, Oliveira FRL. Interdisciplinaridade e intersetorialidade na Estratégia Saúde da Família e no Núcleo de Apoio à Saúde da Família: potencialidades e desafios. Mundo Saúde (Impr.). 2014; 38:129-138.

20. Bispo Júnior JP, Moreira DC. Cuidado colaborativo entre os Núcleos de Apoio à Saúde da Família e as equipes apoiadas. Physis (Rio J.). 2018; 28:e280310.

21. Souza FLD, Chacur EP, Rabelo MRG, Silva LAM, Villela WV. Implantação do Núcleo de Apoio à Saúde da Família: percepção do usuário. Saúde Debate. 2013; 37:233-240.

22. Barros JO, Gonçalves RMA, Kaltner RP, Lancman S. Estratégia do apoio matricial: a experiência de duas equipes do Núcleo de Apoio à Saúde da Família (NASF) da cidade de São Paulo, Brasil. Ciênc. Saúde Colet. 2015; 20:2847-2856.

23. Ministério da Saúde. Núcleo de Apoio à Saúde da Família Brasília: Ministério da Saúde; 2014.

24. Souza TS, Medina MG. Nasf: fragmentação ou integração do trabalho em saúde na APS? Saúde debate 2018 42:145-158.

25. Nascimento AG, Cordeiro JC. Núcleo ampliado de saúde da família e atenção básica: análise do processo de trabalho. Trab Educ Saúde. 2019; 17:e0019424.

26. Spedo SM, Pinto NRS, Tanaka OY. O difícil acesso a serviços de média complexidade do SUS: o caso da cidade de São Paulo, Brasil. Physis (Rio J.). 2010; 20:953972.

27. Ministério da Saúde (MS), Universidade Federal do Rio Grande do Sul (UFRGS). Protocolos de encaminhamento da Atenção Básica para Atenção Especializada Endocrinologia e Nefrologia. Brasília: MS, UFRGS; 2015.

\section{CORRESPONDÊNCIA}

Andreia Marinho Barbosa

Rua Conde d'Eu, 93, apto 602, Santo Amaro,

Recife, PE, Brasil. CEP 50050-470.

E-mail: amb yeshua@yahoo.com.br 